\title{
INEQUALITIES ON REAL ROOTS OF POLYNOMIALS
}

\section{DORU ŞTEFĂNESCU}

\begin{abstract}
We survey the most used bounds for positive roots of polynomials and discuss their efficiency. We obtain new inequalities on roots of polynomials. Then we give new inequalities on roots of orthogonal polynomials, obtained from the differential equations satisfied by these polynomials.
\end{abstract}

Mathematics subject classification (2000): 12D10, 68W30.

Keywords and phrases: Polynomials roots, Inequalities on real roots.

\section{REFERENCES}

[1] A. Akritas, A. Strzeboński, P. Vigklas, Implementations of a new theorem for computing bounds for positive roots of polynomials, Computing, 78, 355-367 (2006).

[2] O. Bottema, Die Nullstellen der Hermitischen Polynome, Nederl. Akad. Wetensch. Proc., 33, 495-503 (1930).

[3] W. H. Foster, I. KRASIKOv, Bounds for the extreme zeros of orthogonal polynomials, Int. J. Math. Algorithms, 2, 307-314 (2000).

[4] J. B. Kioustelidis, Bounds for positive roots of polynomials, J. Comput. Appl. Math., 16, 241-244 (1986).

[5] I. KRASIKov, Nonnegative Quadratic Forms and Bounds on Orthogonal Polynomials, J. Approx. Theory, 111, 31-49 (2001)

[6] A. LAforgia, A monotonic property for the zeros of ultraspherical polynomials, Proc. Amer. Math. Soc., 83, 757-758 (1981),

[7] J.-L. Lagrange, Traité de la résolution des équations numériques, Paris (1798). (Reprinted in Euvres, t. VIII, Gauthier-Villars, Paris (1879).)

[8] E. LAGUERRE, Mémoire pour obtenir par approximation les racines d'une équation algébrique qui a toutes les racines réelles, Nouv. Ann. Math., 2ème série, 19, 161-172, 193-202 (1880).

[9] G. Longchamp, Théormème d'algeèbre, Nouv. Ann. Math, Série 2, 19, 71-74 (1842).

[10] M. MignotTe, D. ŞTEFĂnescu, Polynomials - An algorithmic approach, Springer Verlag (1999).

[11] A. VAN DER SLuIS, Upperbounds for Roots of Polynomials, Numer. Math., 15, 250-262 (1970).

[12] D. ŞTEFĂNESCU, New bounds for the positive roots of polynomials, J. Univ. Comp. Sc., 11, 2125-2131 (2005).

[13] D. ŞTEFĂNESCU, Inequalities on Upper Bounds for Real Polynomial Roots, in Computer Algebra in Scientific Computing, 284-294, LNCS 4194 (2006).

[14] G. Szegö, Orthogonal Polynomials, Proc. Amer. Math. Soc. Colloq. Publ., vol. 23, Providence, RI (2003).

[15] S. C. VAN VeEN, Asymptotische Entwicklung un Nullstellenabschätzung der Hermitische Funktionen, Nederl. Akad. Wetensch. Proc., 34, 257-267 (1931).

[16] C. K. YAP, Fundamental problems of algorithmic algebra, Oxford University Press (2000). 